\title{
Localization Techniques for Wireless Sensor Networks
}

\author{
Aditi Shrivastava \\ Mtech. O.C.T Bhopal (M.P.) India
}

\author{
Priya Bharti \\ Dept. of CSE O.C.T. Bhopal (M.P.) India
}

\begin{abstract}
Wireless sensor networks (WSNs) have recently emerges as promising technology in wireless communication field and gained special attention by research groups. It uses small and cheap gadgets with low energy requirements and limited on board computing resourceswhich communicates with each other's or base stations without any pre-defined infrastructure. The property of being infrastructure less makes it suitable in distinctive application situations including remotemonitoring, disaster management, military applications and biomedical health observing devices. In many of these applications, node localization is unavoidably one of the important system parameters for example in target tracking if the nodes are not able to obtain the accurate location information, the related task cannot be performed.It is also helpful in routing, network coverage and quarry management of sensors. In general the localization techniques are ordered into two general classifications: range based and range free. In this paper, we discussed the various localization algorithms with their applicable areas, requirements and limitations. Moreover, on conclusion we compare these localization algorithms and analyze the future research directions for the localization algorithms in WSNs.
\end{abstract}

\section{Keywords}

Wireless Sensor Networks, Localization Techniques.

\section{INTRODUCTION}

Recent developments in semiconductor technology which makes feasible to design large complex circuits into a single integrated circuits (IC) with low power consumption and small form factor opens up the new applicable areas for wireless sensor networks. A Wireless sensor network (WSN) is structured by many little, cheap gadgets called sensors which have limited memory, power and processing capabilities. These sensors are conveyed to sense the physical characteristics of the surrounding environment, for example, temperature, light and contamination. However depending upon the requirements the sensing parameters of sensors can be changed for extensive variety of uses, for example, remote sensing, disaster management, patient tracking, and militaryobservations. In a significant number of these applications, area localization is helpful or even fundamental requirement. In fact, without knowing the position of sensor node, gathered information is valueless. The localization of sensors can be executed by diverse behavior. A straightforward arrangement is to outfit every sensor node with a GPS beneficiary that can absolutely furnish the sensor nodes with their precise position.On the other hand, including the GPS to all nodes in the

Wireless sensor network is not preferable because of its high cost, high power consumption and environment obligation.Furthermore, the GPS fails in underground applications. Because of such limitations other localization techniques are utilized one of them is self-localization, in which sensor nodes can appraise their position by utilizing different localization revelation algorithms. These algorithms utilize a couple of special nodes, called beacon nodes, which are expected to know their own particular location (through manual setup or GPS). These beacon nodes (or anchor nodes) give position data, as reference point, for the other nodes, which can use these position data from different close-by reference beacon nodes to estimate their own positions. Whatever remains of this paper is sorted out as take after. In area 2, order of localization calculations is given. In area 3 , relative investigation of localization calculations is talked about. Area 4 closes the paper and blueprints future conceivable exploration.

\section{LOCALIZATION TECHNIQUES}

Practically all current localization plans comprise of two stages:

\subsection{Distance/Angle Estimation}

In distance/angle estimation, the most widely recognized range estimation procedures used to estimate distance or angle between two sensor nodes are TDOA (Time Difference Of Arrival , TOA (time of landing), RSSI (Received Signal Strength Indicator), AOA (angle of entry) and Hop-tally.

\subsection{Position Calculation}

In position calculation, the position of the obscure node is evaluated focused around the accessible data of distance or angle and positions of references nodes. The ordinarily utilized strategies incorporate lateration, triangulation, bounding box, probabilistic approach and fingerprinting.

Majority of literatures available on node localization focused around a few unique criteria, for example, reliance of the range estimations (i.e. range-based localization or without range localization); distributed or centralized position estimation; with or without a base (anchor based localization or anchor free localization). As per the reliance of range estimation systems, localization calculations can be characterized into two primary classes: range-based techniques and range-free techniques.

\section{RANGE-BASED LOCALIZATION TECHNIQUES}

This group belongs to techniques which utilizes range measurement for location calculation. As indicated by the way of utilizing the range estimation methods. Range-based techniques uses range measurements such as time of arrival (ToA), angle of arrival (AoA), received signal strength indicator (RSSI), and time difference of arrival (TDoA) to measure the distances between the nodes in order to estimate the location of the sensors. The range based technique can further divided into anchor based or anchor less technique.

\subsection{Anchor Based Techniques}

Anchor nodes are also known as reference nodes which have well defined information about its location. Hence in anchor based technique other nodes utilize anchor nodes to estimate their positions. In such calculations, a small amount of the nodes must be anchor nodes or possibly a minimum number of anchor nodes are needed for satisfactory results. No less than three non-collinear anchor nodes for 2-dimensional 
spaces and four non-coplanar anchor nodes for 3-dimensional spaces are needed.

\subsubsection{Time of Arrival (ToA)}

During the localization process, the sensor detects the time of arrival measurement of the anchor node signals at its receiver based on particular signal features (e.g., preamble) transmitted by the source node. Given an LOS (line of sight) propagation path, the time of arrival measurement at sensor node $y$ can be modeled as

$$
t_{j}=\frac{1}{c} \sqrt{\sum_{i=1}^{N}\left(x_{i}^{j}-y\right)^{2}}+t_{0}+n_{j}, \ldots \ldots \ldots \ldots .
$$

where $c$ is the speed of light, $N$ denotes the dimensions, $x_{i}^{j}$ is the value of $i^{\text {th }}$ dimention of $j^{\text {th }}$ anchor node, $t_{0}$ is the unknown time instant at which the source transmitsthe signal to be measured, and $n_{j}$ is the additive measurementnoise (error) with zero mean for $j^{\text {th }}$ anchor node. Note that the sensors just estimate the sign TOA $t_{j}$ rather than the sign propagation time. To estimate the propagation time, the source must collaborate by synchronizing its "time of transmission" with the sensors, or it must encode a time stamp inside the transmitted frame to direct the sensors what $t_{0}$ is. Without such time synchronization or time stamp, the TOA estimation comprises of an extra unknown $t_{0}$.

Without any other prior assumptions on the statistics of the TOA measurements, a least square (LS) estimator can be used for the source localization problem, i.e.

$$
\left(\hat{y}, \widehat{t_{0}}\right)=\underset{y_{i}, t_{0}}{\arg \min } \sum_{j=1}^{M}\left(t_{j}-\frac{1}{c} \sqrt{\sum_{i=1}^{N}\left(x_{i}^{j}-y_{i}\right)^{2}}-t_{0}\right)^{2} \ldots
$$

Using optimization techniques, we can implement direct optimization by searching for the optimum coordinates of $y$ and $t_{0}$ that minimize (2). Some of the optimization techniques which can be proffered according to problem characteristics are maximum likelihood (ML) and least square (LS) however recently developed evolutionary algorithms like genetic algorithm (GA), artificial bee colony (ABC) optimization and practical swarm optimization (PSO) can also be utilized

\subsubsection{Time Difference of Arrival (TDoA)}

Because The TOA model needs to estimate both $y$ and $t_{0}$ jointly which makes the optimization problem rather challenging as a multidimensional search problem also that the unknown $t_{0}$ is not of direct interest in source localization. Hence a modified approach is proposed in which the resulting TOA measurement are preprocessed through pairwise subtraction to generate the measurement for time difference of arrival based localization, independent of $t_{0}$, and known as Time Difference of Arrival (TDoA).

In order to obtain the time-difference of arrival, a simple preprocessing calculations of the TOA measurement is given by

$$
\Delta_{i j}=t_{i}-t_{j}=\frac{1}{c}\left(\sqrt{\sum_{k=1}^{N}\left(x_{k}^{i}-y_{i}\right)^{2}}-\sqrt{\sum_{k=1}^{N}\left(x_{k}^{j}-y_{i}\right)^{2}}\right)+n_{i}
$$$$
-n_{j},
$$

Where $\Delta_{i j}$ is the TDoA measurement.

Now it's clear from equation (3) that calculation for timedifference removes the unknown parameter $t_{0}$. However detailed study of equation (3) revealsthat there are two issues for this transforming. Firstly, we note that the terms $n_{i}-n_{j}$ in (3) are no more independent, hencethe terms $n_{i}-n_{j}$ and $n_{m}-n_{j}$ are related since they have in common term $n_{j}$. Also, in correlation with the uncorrelated noise in the first TOA model (1), the subtraction likewise strengthens the noise in TDOA by exactly by factor of two. Hence, the preprocessing for getting TDOA may cause the performance degradation which should be avoided.

\subsubsection{Angle of Arrival (AOA)}

The localization processare focused around a fundamental technique where sensor node noting the times and angle when it gets the signals from different anchor nodes, and then calculatestheir location by triangulation. Denote the times at which asensor node $S_{i}$ receives the beacons signals at instantst $t_{1}, t_{2}, t_{3}$, and $t_{4}$ from anchor $\operatorname{nodes} A_{1}, A_{2}, A_{3}$ and $A_{4}$ (since only four anchor nodes are sufficient for 3 dimensional space) respectively. Because the sensor nodes have no time synchronization mechanism with the beacon nodes, the absolute values of these times cannot be utilized,instead the time diff erence of arrivals can be translated to angular values by using equation (4):

$$
\alpha=\phi-\omega \tau_{1}, \beta=\phi-\omega \tau_{2}, \gamma=\phi-\omega \tau_{3},
$$

where $\omega$ is the angular velocity of the anchor nodes field rotation, $\tau_{1}=t_{2}-t_{1}, \tau_{2}=t_{3}-t_{2}$, and $\tau_{3}=t_{4}-t_{3} \quad$ are time differences. Any two angles chosen from $\alpha, \beta$, and $\delta$ can then be used to solve for the location of the $S_{i}$ using trigonometry. For instance, using the values of $\alpha$ and $\beta$, we get:

$$
\gamma=\tan ^{-1}\left[\frac{\cos (\beta)-S \sin (\alpha)}{S \cos (\alpha)-\sin (\beta)}\right], \text { where } S=\frac{L \sin (\beta)}{W \sin (\alpha)}
$$

$L$ and $W$ are the length and the width of the rectangle created by the four anchor nodes.

$$
Y=L\left(\frac{\sin (\gamma-\alpha)}{\sin (\alpha)}\right)
$$

With these, the location of $S_{i}\left(X_{p}, Y_{p}\right)$ is given by

$$
X_{p}=Y \cos (\gamma), Y_{p}=M-Y \sin (\gamma),
$$

Note: In the presented system the anchor nodes are assumed to be equipped with rotating transmission beam whereas the sensor nodes having Omni directional antenna however the opposite of assumption can also be used.

There are some limitations with this location discovery technique, for example if there is multipath reflection the $S_{i}$ receivesdifferent multipath reflected signals from single anchor node. This insists for specific requirements such that very narrow (ideally zero) beam width of the anchor antenna.

\subsubsection{Received Signal Strength Indicator (RSSI)}

In this technique sensor nodes uses the Received Signal Strength (RSS) for the measurement of distance from anchor nodes and then based on these measurements they estimate theirlocations.

The relation between the RSSI and distance is presented by Log Distance Path Loss Model which is a fundamental 
method of estimating path loss as a function of distance between the nodes. The model is generally expressed as following equation.

$$
\begin{array}{rl}
L(d B)=P_{0}+10 & * n * \log _{10}\left(\frac{d}{d_{0}}\right) \\
& +X_{\sigma}, \ldots \ldots \ldots \ldots \ldots \ldots \ldots
\end{array}
$$

where $n$ is the path loss exponent, $d$ is the distance between transmitter and receiver, $X_{\sigma}$ is a Gaussian random variable with standard deviation $\sigma$ and $P_{0}$ is the received power at reference distance $d_{0}$

The RSSI is calculated by equation (8)

$$
\begin{array}{r}
R S S I=-10 * n * \log _{10}(d) \\
+A, \ldots \ldots .
\end{array}
$$

Where $n$ is propagation exponent, $d$ is the distance from the sender and $A$ is the received signal strength at one meter of distance.

Ones the distance is calculated the node positions is estimated by minimizing the sum of the discrepancies between the estimated distance between the nodes and the measured one (Minimum Least Square algorithm).

$$
(\hat{y})=\underset{y_{i}}{\arg \min } \sum_{j=1}^{M}\left(\sqrt{\sum_{i=1}^{N}\left(x_{i}^{j}-y_{i}\right)^{2}}-\widehat{d_{J}}\right)^{2}, \ldots
$$

where $d_{j}$ is the distance from $j^{\text {th }}$ anchor node and $\hat{y}$ is the coordinates of node to be estimated.

A disadvantage to anchor-based calculations is that an alternate positioning system is obliged to accurately defining the anchor node positions. Hence, if the other positioning system is inaccessible, for example, for GPS-based anchors spotted in regions where there is no reasonable perspective of the sky, the calculation may not work legitimately. An alternate disadvantage to anchor-based calculations is that anchor nodes are costly as they typically oblige a GPS recipient to be mounted on them. Subsequently, algorithms that requires numerous anchor nodes are not financially preferable. On the non-GPS anchors where the location information is hard-coded into anchor nodes careful placement of anchor nodes is necessary, which may be very expensive or even impossible in many cases.

\subsection{Anchor Free Techniques}

The most common Range-based localization algorithms that don't require anchor nodes, like ABC (Assumption Based Coordinates) algorithm are based on RSSI measurements to determine the inter node distances. In order to calculate the inter-node distances,this scheme first selects four in range sensor nodes and assigns them coordinates. The coordinates of other nodes are incrementally calculated using the distances from atleast four nodes with already calculated coordinates.

The ABC algorithm does not require complicate calculation, hence considerably simpler than Range-based algorithms however the localization accuracy is quite low especially for widespread networks. Also the algorithm accumulates the error in every iteration which results in gradual degradation of positioning accuracy which decreases from the node which started algorithm hence in real network the complete graph realization is not guaranteed.
Furthermore, even if one measurement is corrupted by noise, the algorithm can lead to completely incorrect nodes localizations.

\section{RANGE-FREE LOCALIZATION TECHNIQUES}

Range-free techniques use connectivity information among neighboring nodes to properly estimate the node's location hence range-free techniques do not require any additional hardware and use nearby nodes information to estimate the location of the nodes in the network, although these techniques have limited precision.

Like Range-based algorithm the range free algorithms also divided into anchor based or anchor less types.

\subsection{Anchor Based Algorithm}

\subsubsection{Centroid}

The Centroid scheme was proposed by Bulusu et al. in [2]. This localization scheme requires a set of anchor nodes $\left(A_{i}, 1 \leq i \leq n\right)$,such that the transmission range of these nodes creates common regions of availability, exist in the placement area of the WSN. The main idea is to calculate the center of gravity of all anchor nodes by treating the anchor nodes, located at $\left(X_{i}, Y_{i}\right)$, as point masses $m_{i}$. In the most general form the coordinates of center of gravity of the centroid of $n$ point masses $m_{i}$ are given by:

$$
\left(X_{G}, Y_{G}\right)=\left(\frac{\sum_{i=1}^{n} m_{i} X_{i}}{\sum_{i=1}^{n} m_{i}}, \frac{\sum_{i=1}^{n} m_{i} Y_{i}}{\sum_{i=1}^{n} m_{i}}\right)
$$

which, for equal masses $m_{i}$ simplifies to:

$$
\left(X_{G}, Y_{G}\right)=\left(\frac{\sum_{i=1}^{n} X_{i}}{n}, \frac{\sum_{i=1}^{n} Y_{i}}{n}\right),
$$

A node $N_{k}$ computes its location as the average of all the anchor nodes $A_{i}$ it has heard from with a connectivity higher than a threshold.

$$
\left(X_{k}, Y_{k}\right)=\left(\frac{\sum_{i=1}^{N} X_{i}}{N}, \frac{\sum_{i=1}^{N} Y_{i}}{N}\right),
$$

where $N$ is the number of anchors with a higher connectivity than the threshold.

\subsubsection{Area Based}

\subsubsection{Area-Based Point-In-Triangulation Test (APIT) localization scheme}

It expect that a little number of nodes, called anchors, are equipped with high-power transmitters and knows their area, by means of GPS or some other component. Utilizing beacons from these anchors, it utilizes a region based methodology to perform area estimation by segregating area into triangular region between anchor nodes as indicated in Figure 1. A node's vicinity inside or outside of these triangular region permits a node to restricted down the region in which it can conceivably reside. By using diff erent mixes of anchors, the measure of the estimated territory in which a node resides can be decreased, to give a decent location estimate. 


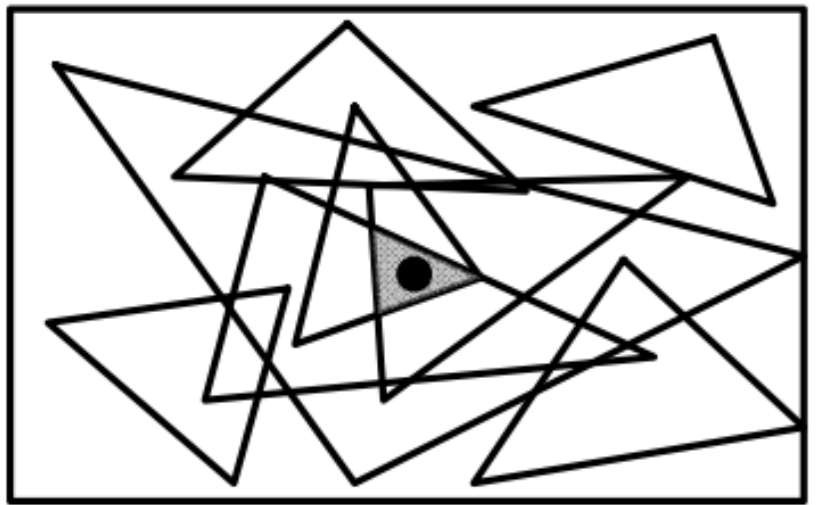

Figure 1: Area-based APIT Algorithm triangular region formation

The theoretical method used to narrow down the possible area in which a target node resides is called the Point-InTriangulation Test (PIT). For three given anchors: $A\left(a_{x}, a_{y}\right), B\left(b_{x}, b_{y}\right), C\left(c_{x}, c_{y}\right)$, the Point-In-Triangulation test determines whether a point $\mathrm{M}$ with an unknown position is inside triangle $\triangle A B C$ or not. It repeats this PIT test with different anchor combinations until all combinations are exhausted or the required accuracy is achieved. At this point, it calculates the center of gravity (COG) of the intersection of all of the triangles in which a node resides to determine its estimated position.

\subsubsection{Secure range-independent localization (SeRLoc)}

Serloc is an alternate approach for region based range-free localization. The algorithm uses two sorts of nodes: ordinary nodes and locators (i.e., anchors). Ordinary nodes are furnished with omnidirectional reception antennas, while anchors are outfitted with directional antennas and their locations are known. In Serloc, a sensor estimates it location on the basis of data transmitted by the locators. Figure 6 demonstrates the primary thought, with node $N_{k}$ inside radio range to locators $A_{1}, A_{2}$ and $A_{3}$ :

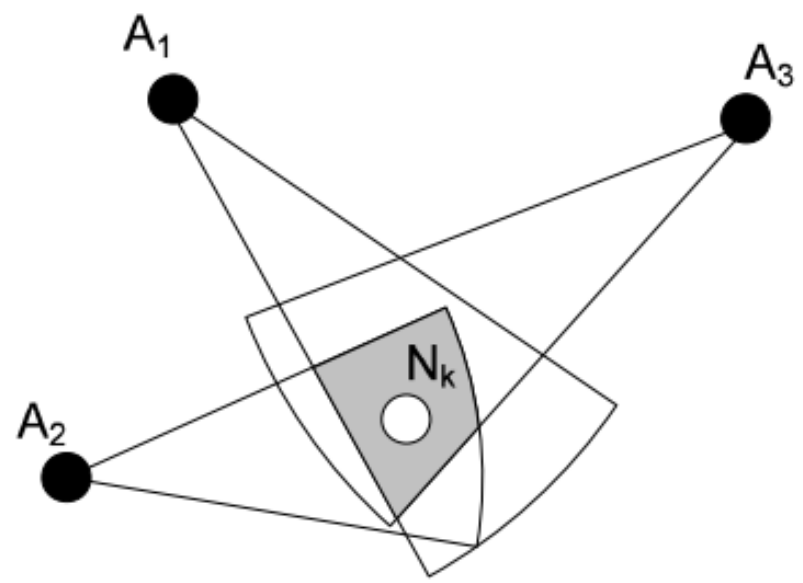

Figure 2: Secure range-independent localization

Serloc locates the sensor nodes in four steps. To begin with, a locator transmits directional beacons inside a segment. Each one beacon contains the locator's position and the angles of the division limit lines. A typical node gathers the beacons from all locators it listens. Second, it decides a rough search region inside which it is spotted by using the directions of the locators listened. Third, it processes the covering segment region utilizing a dominant part vote plan. At last, Serloc decides a node area as the center of gravity of the covering district.

\subsubsection{Ad-Hoc Positioning System}

The Ad-Hoc Positioning System (APS) uses the hop-by-hop propagation of distances to known anchor nodes. After obtaining distance estimates to three or more anchors, a sensor node employs a multilateration for iteratively improving its location estimation. This algorithm mainly differ from the previous algorithms on the basisthat how a sensor node $N_{j}$ estimates its distance $\left(d_{j i}\right)$ to an anchor $A_{i}$. The steps of the APS localization scheme algorithm are the following:

- $\quad$ Each anchor node $A_{i}$ initiates a flood of the network by broadcasting a packet containing its position and a counter with the initial value set to one.

- $\quad$ Each sensor node $N_{j}$ keeps track of the shortest path (in terms of radio hop counts, $h_{j, A_{i}}$ ) to an anchor $A_{i}$ from which it has received a beacon. The authors also propose four methods for propagating the distances from anchors to sensor nodes: DV-Hop, DV-Distance, Euclidian and Coordinate. The method that does not assume ranging, DV-Hop is described below. An example of the DV-Hop scheme is shown in Figure 3. At the end of this phase, node $N_{j}$ knows that it is 3 hops, 2 hops and 1 hop from $A_{1}, A_{2}$ and $A_{3}$, respectively.

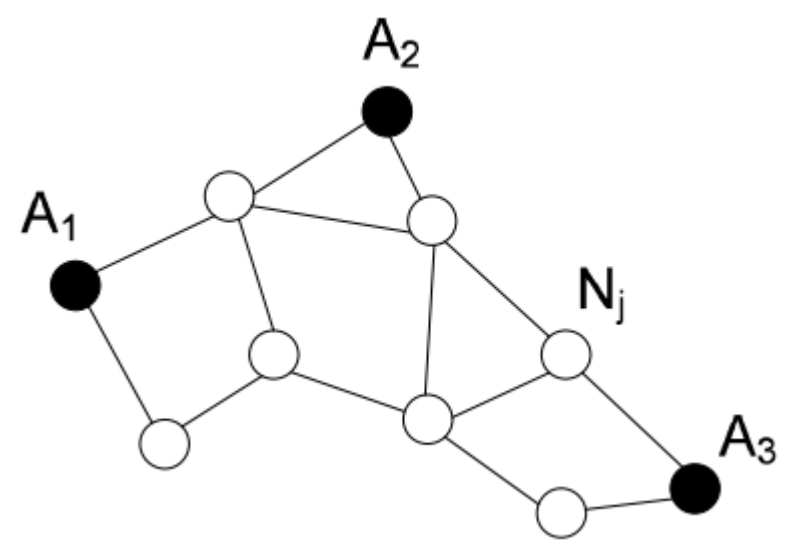

Figure 3: The DV-Hop localization scheme

Once an anchor node $A_{i}$ obtains distances to other anchors, it computes a correction factor $c_{i}$ (the estimated 1 radio hop Euclidian distance), which it propagates in the network. Corrections are propagated through controlled flooding, i.e., after a node receives and forwards the first correction, it will stop forwarding subsequent corrections. The correction factor is computed as follows:

$$
c_{i}=\frac{\left(\sum \sqrt{\left(x_{i}-x_{j}\right)^{2}+\left(y_{i}-y_{j}\right)^{2}}\right)}{\sum h_{i}}
$$

for all anchors $A_{j} \neq A_{i}$ from which it has received a beacon (anchor $A_{j}$ is positioned at $\left(x_{j}, y_{j}\right)$ and $h_{i}$ is the number of hops between the sensor node and anchor $A_{i}$ ).

A least square method (the authors used the Householder method) is employed for solving the non-linear system of equations: 


$$
\left[\begin{array}{c}
\Delta \rho_{1} \\
\Delta \rho_{2} \\
\ldots \\
\Delta \rho_{n}
\end{array}\right]=\left[\begin{array}{cc}
\hat{1}_{1 x} & \hat{1}_{1 y} \\
\hat{1}_{2 x} & \hat{1}_{2 y} \\
\ldots & \ldots \\
\hat{1}_{n x} & \hat{1}_{n y}
\end{array}\right]\left[\begin{array}{l}
\Delta x \\
\Delta y
\end{array}\right]
$$

where $\Delta \rho_{i}=\widehat{\rho}_{l}-\rho_{i}, \widehat{\rho}_{l}$ and $\rho_{i}$ are the estimated and the real distances between a sensor node and an $\operatorname{anchor} A_{i}, \hat{1}_{i x}$ is the unit vector of $\widehat{\rho}_{l}$ in the $x$ direction and $\Delta x$ and $\Delta y$ are the corrections in the position estimatefor the node $N_{j}$.

\subsection{Anchor-Free Solutions}

\subsubsection{Spotlight}

The main idea of the Spotlight localization system is to generate controlled events in the field where the sensor nodes are deployed. An event could be, for example, the presence of light in an area. Using the time when an event is perceived by a sensor node and the spatio-temporal properties of the generated events, spatial information (i.e. location) regarding the sensor node can be inferred. The system architecture for the Spotlight localizationsystem is shown in Figure 4.

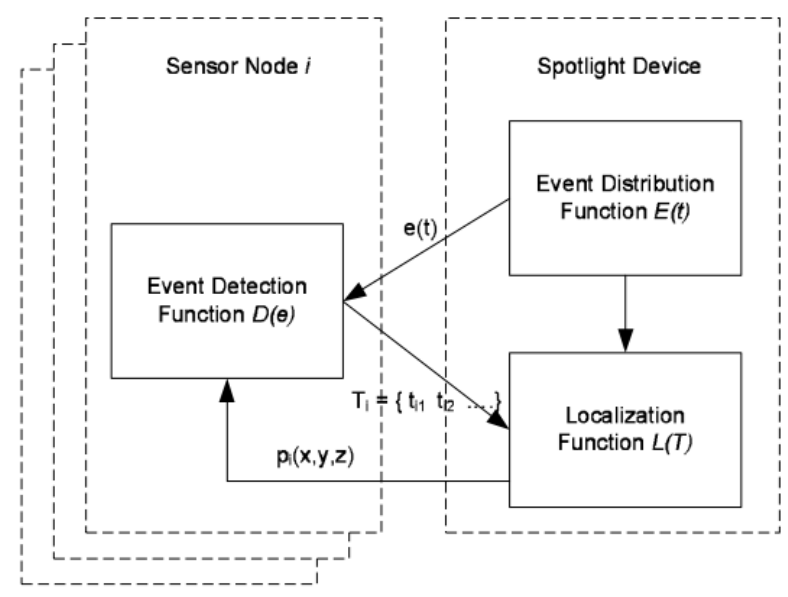

Figure 4: Spotlight system architecture

With the support of these three functions, the localization process goes as follows:

- A Spotlight device distributes events $e(t)$ in the space $A$ over a period of time.

- During the event distribution, sensor nodes record the time sequence $T_{i}=\left\{t_{i 1}, t_{i 2}, \ldots, t_{i n}\right\}$ at which they detect the events.

- After the event distribution, each sensor node sends the detection time sequence back to the Spotlight device.

- The Spotlight device estimates the location of a sensor node $i$, using the time sequence $T_{i}$ and the known $E(t)$ function.

The Event Distribution Function $E(t)$ is the core technique used in the Spotlight system and the authors propose three designs for it, with diff erenttradeoff s/costs. These designs are Point Scan, Line Scan and Area Cover.

\subsubsection{Walking-GPS}

In many applications it is envisioned that WSN will be deployed from Unmanned Aerial Vehicles. In the meantime, manual deployments have been prevalent and the employed localization solutions have used some variant of associating the sensor node ID with prior knowledge of that ID's position in the field.

In Walking GPS, the deplorer (either person or vehicle) carries a GPS device that periodically broadcasts its location. The sensor nodes being deployed, infer their position from the location broadcast by the GPS device. The proposed solution is simple, cost eff ective and has very little overhead. In the Walking GPS architecture the system is decoupled into two software components: the GPS Node and the Sensor Node. The GPS node is connected to a GPS device, and outputs its location information at periodic intervals. The Sensor node component runs on all sensor nodes in the network. This component receives the location information broadcast by the GPS nodes and infers its position from the packets received. The proposed architecture pushes all complexity derived from the interaction with the GPS device to a single node, the GPS Node, and to significantly reduce the size of the code and data memory used on the sensor node. Through this decoupling, a single GPS Node is sufficient for the localization of an entire sensor network, and the costs are thus reduced. A relatively simple design for the GPS Node would have been to periodically broadcast the actual GPS location received from the GPS device. In order to reduce the overhead incurred when exchanging data containing global GPS coordinates, the Walking GPS system uses a local, Cartesian, coordinate system. The conversion between coordinate systems is performed by the GPS node. A local coordinate system of reference is better suited for WSN, than a global coordinate system. The localization scheme that makes use of the Walking GPS solution has two distinct phases:

1. The first phase is during the deployment of the sensor nodes. This is when the Walking GPS solution takes place. The deployer has a GPS-enabled mote attached to it; the GPSenabled mote periodically beacons its location; the sensor nodes that receive this beacon infer their location based on the information present in this beacon.

2. The second phase is during the system initialization. If at that time, a sensor node does not have a location, it asks its neighbors for their location information. The location information received from neighbors is used in a triangulation procedure by the requester, to infer its position. This second phase enhances the robustness of the scheme.

\section{CONCLUSION}

Despite the fact that WSNs are a relatively new concept, there are a number of different localization approaches, each with an attention on particular situation and/or application has already presented. In this paper, we examine and analyzed some of the recent localization algorithms, and concluded that among all considered methods, this relative investigation led us to infer that every calculation has its own advantages and disadvantages and none is completely the best. In general, the range-based systems are either no-cost effective regarding equipment expense, or limited by to natural conditions. Interestingly, the range-free strategies are uncertain and effectively influenced by node density. Then again, it has lower accuracy. As we known exactness is the most imperative key for localization execution. Among the plans dissected in this paper, range-based techniques look encouraging, considering that the cost of positioning equipment's are decreasing continuously making this technique a successful answer for the localization in wireless sensor networks. However in future the range estimation procedures between anchor nodes and sensor nodes can be developed. 


\section{REFERENCES}

[1] Guoqiang Mao, Barıs, Fidan and Brian D.O. Anderson "Wireless Sensor Network Localization Techniques", Computer Networks Volume 51, Issue 10, 11 July 2007, Pages 2529-2553.

[2] AsisNasipuri and Kai $\mathrm{Li}$ "A Directionality based Location Discovery Scheme for Wireless Sensor Networks", WSNA '02 Proceedings of the 1st ACM international workshop on Wireless sensor networks and applications Pages 105-111 ACM New York, NY, USA (C)2002.

[3] Masashi Sugano, Tomonori Kawazoe, Yoshikazu Ohta, and Masayuki Murata "Indoor Localization System Using RSSI Measurement Of Wireless Sensor Network Based On Zigbee Standard", Wireless and Optical Communications, IASTED/ACTA Press, (2006).

[4] RongPeng and Mihail L. Sichitiu "Angle of Arrival Localization for Wireless Sensor Networks", Sensor and Ad Hoc Communications and Networks, 2006. SECON '06. 2006 3rd Annual IEEE Communications Society on (Volume:1) 28-28 Sept. 2006 Page(s): 374 - 382

[5] MohitSaxena, Puneet Gupta, BijendraNath Jain "Experimental Analysis of RSSI-based Location Estimation in Wireless Sensor Networks", Communication Systems Software and Middleware and Workshops, 2008. COMSWARE 2008. 3rd International Conference on 6-10 Jan. 2008 Page(s): 503 - 510.

[6] Ravindra S and Jagadeesha S N "Time of Arrival Based Localization In Wireless Sensor Networks: A Linear Approach”, Signal \& Image Processing: An International Journal (SIPIJ) Vol.4, No.4, August 2013

[7] Kuo-Feng Ssu, Chia-Ho Ou, and Hewijin Christine Jiau, "Localization with Mobile Anchor Points in Wireless Sensor Networks", IEEE Transactions on Vehicular Technology, Vol. 54, No. 3, May 2005.

[8] F. Viani, L. Lizzi, P. Rocca, M. Benedetti, M. Donelli, and Andrea Massa "Object Tracking through RSSI Measurements in Wireless Sensor Networks", May 2008, University of Trento.
[9] Jan Blumenthal, Frank Reichenbach, Dirk Timmermann "Minimal Transmission Power vs. Signal Strength as Distance Estimation for Localization in Wireless Sensor Networks", International Workshop on Wireless Ad-hoc and Sensor Networks (IWWAN 2006), New York.

[10] Guoqiang Mao, BarısFidan, Brian D.O. Anderson "Wireless sensor network localization techniques", Computer Networks 51 (2007) 2529-2553.

[11] Lingxuan Hu, David Evans "Localization for Mobile Sensor Networks”, MobiCom'04, Sept. 26.-Oct. 1, 2004, Philadelphia, Pennsylvania, USA.

[12] RaduStoleru, Tian He and John A. Stankovic "RangeFree Localization", Secure Localization and Time Synchronization for Wireless Sensor and Ad Hoc Networks Advances in Information Security Volume 30, 2007, pp 3-31.

[13] AsmaMesmoudi, Mohammed Feham, Nabila Labraoui "Wireless Sensor Networks Localization Algorithms: A Comprehensive Survey", International Journal of Computer Networks \& Communications (IJCNC) Vol.5, No.6, November 2013

[14] A. Faheem, R. Virrankoski and M. Elmusrati "Improving RSSI based distance estimation for 802.15.4 wireless sensor networks", Wireless Information Technology and Systems (ICWITS), 2010 IEEE International Conference on Aug. 28 2010-Sept. 32010 Page(s): 1 - 4.

[15] Hady S. AbdelSalam, Stephan Olariu "Towards Enhanced RSSI-Based Distance Measurements and Localization in WSNs", INFOCOM Workshops 2009, IEEE 19-25 April 2009 Page(s): 1 - 2.

[16] EnyangXu, IEEE, Zhi Ding, and SouraDasgupta "Source Localization in Wireless Sensor Networks from Signal Time-of-Arrival Measurements", IEEE Transactions on Signal Processing, Vol. 59, No. 6, June 2011.

[17] Khalid K. Almuzaini, Aaron Gulliver "Range-Based Localization in Wireless Networks Using Density-Based Outlier Detection", Wireless Sensor Network, 2010, 2, 807-814. 